\title{
The Fine Structure of the Terminal Branches of the Hepatic Arterial System of the Rat '
}

\author{
WILLIAM E. BURKEL \\ Department of Anatomy, The University of Michigan, \\ Ann Arbor, Michigan
}

\begin{abstract}
The fine structure of the smallest branches of the hepatic arterial system of the rat was studied with the light and electron microscope by means of alternate thick and thin serial sections.

The terminal arborizations of the hepatic artery closely follow the pattern of the portal vein and give rise to a dense network of capillaries in the periportal connective tissue which closely surrounds the bile ducts. These periductal capillaries end by joining (a) interlobular veins, (b) terminal distributing veins, (c) sinusoids directly or, (d) sinusoids in common with branches of the portal vein. The capillaries arising from larger arterioles have well developed precapillary sphincters at their origins, while those arising from terminal arterioles have less prominent smooth muscle cuffs. There are no smooth muscle sphincters at the terminal ends of the capillaries where they join branches of the portal vein or sinusoids. Large endothelial cells usually guard these junctions and frequently their nuclei bulge into the lumens of the vessels and may close them off. Occasionally unmyelinated nerves with vesicles about $500 \AA$ in diameter, some of which are granulated, are in close proximity to the endothelial cells of the capillaries. Endothelial cells also guard the beginnings of sinusoids originating from the portal vein. The sinusoids arising from periductal capillaries are identical in structure to those directly off from the portal vein. Initially the sinusoids from both types of vessels are like capillaries, with a continuous basement membrane around them and an unfenestrated endothelium. A short distance into the parenchyma they lose their basement membrane, become fenestrated and are typical sinusoids.
\end{abstract}

The vasculature of the liver has been studied extensively during the three centuries since the time of Glisson. Although a great deal of work has been done, some of the finer details of the relations of the hepatic arterial system to the portal venous system are still obscure.

The interconnections between the portal vein and hepatic artery have been demonstrated using histological sections, wax reconstructions, injections, injection-corrosion methods, roentgenography and transillumination of living specimens. Recent studies by Hase and Brim ('66) and Mitra ('66) fairly well summarize the present understanding of the distribution of the hepatic artery and its terminations as seen in static studies. The functional microcirculation of the liver, however, is a dynamic system with higher pressure hepatic arterial vessels interconnected with lower pressure portal veins and sinusoids and it is best studied in living animals. This has been done extensively by transillumination (Knisely, '36; Block, '40, '55; Wakim and Mann, '42; Wakim, '44; Irwin and Mac-
Donald, '53; Knisely et al., '57; McCuskey, '66). These studies have shown, in addition to the types of connections between the hepatic artery and portal vein, numerous sphincter-like structures which control the flow of blood between the two systems. These "sphincters" are assumed to be contractile reticulo-endothelial or endothelial cells (McCuskey, '66), but their finer structure is unknown.

Fine structure studies of the liver have concentrated mainly on the parenchymal cells, sinusoids, and bile ducts. No definitive work has been done on the hepatic arterial system, its connections to the portal vein and sinusoids, or to the structure of sinusoids arising from terminal branches of the hepatic artery. The structure of sinusoids originating exclusively from portal veins has been studied (Burkel and Low, '66).

The work reported here demonstrates the fine structure of the terminal arborizations

\footnotetext{
Received June 2, '69. Accepted Feb. 12, '70.

1 Supported by a grant from the Faculty Research Fund, Horace H. Rackham School of Graduate Studies, The University of Michigan.
} 
of the hepatic artery and their connections to the portal vein and sinusoids. Particular attention was given to the junctions between the arterial and venous systems and to the areas of the apparent sphincters.

\section{MATERIALS AND METHODS}

Twenty healthy, young adult, albino rats were anesthetized with $3.5 \%$ chloral hydrate ( $1 \mathrm{ml} / 100 \mathrm{gm}$ of rat) and perfused through the heart with glutaraldehyde (Sabatini et al., '63) or gluaraldehydeformaldehyde (Karnovsky, '65) using a modification of the technique of Palay et al. ('62). Perfusion continued until a volume of fixative (in $\mathrm{ml}$ ) equal to, or greater than the weight of the animal had been used. Liver tissue was then removed, minced into blocks approximately $2 \times 2 \times$ $1 \mathrm{~mm}$ in size, washed in buffer and postfixed for two to three hours in $1 \% \quad \mathrm{OsO}_{4}$ containing the same type of buffer used in the perfusion. The tissue was dehydrated in a graded series of alcohols containing 1\% phosphotungstic acid. Embedment was in Epon 812 (Luft, '61) with polmerization at $60^{\circ} \mathrm{C}$ for 24 hours.

Thick sections $(1-2 \mu)$ of the entire face of the embedded tissue blocks were then made for light microscopy and stained at $80^{\circ} \mathrm{C}$ with either $1 \%$ toluidine blue in $1 \%$ borax, or Paragon Multiple Stain for Frozen Sections, ${ }^{2}$ also containing $1 \%$ borax. After examination of the thick sections, the blocks were trimmed for ultramicrotomy so that they included a suitable portal area. Finally, alternating thin (for electron microscopy) and thick (for light microscopy) serial sections of the selected portal areas were made in order to follow the branches of the hepatic artery. Thin sections were mounted on single hole grids which were covered with formvar membranes and stained with lead citrate (Reynolds, '63). The thick sections were stained as above.

\section{OBSERVATIONS}

The hepatic artery winds through the portal space in close association with the portal vein, bile ducts and lymphatic vessels giving off numerous branches along its course. As the portal vein branches and becomes smaller the artery does also, until deep within the liver it is represented only by arterioles and capillaries. Two or three arterial branches usually accompany a portal vein of about $600-700 \mu$ in diameter (fig. 2). One of the branches (figs. 2,3) is generally larger and it gives rise to the smaller ones (fig. 4). The small branches in turn give rise to rich network of capillaries (fig. 7) found in the portal space.

On the bases of their size and the structural makeup of their vascular walls, it is convenient to describe four different types of vessels forming the terminal parts of the hepatic arterial system. ${ }^{3}$ Vessels approximately 50-100 $\mu$ in diameter and with a wall 10-15 $\mu$ thick are considered to be arterioles. Terminal arterioles are 10-50 $\mu$ in diameter and have a wall about $5 \mu$ thick. Vessels with diameters less than $10 \mu$ are considered to be capillaries. The transitional vessels between terminal arterioles and capillaries, with lumens of about $10 \mu$, but with walls about $5 \mu$ thick are considered to be precapillaries.

\section{Arterioles}

Vessels with a caliber of 50-100 $\mu$ are considered to be arterioles. A large arteriole (about $100 \mu$ ) typically accompanies a portal vein of about $500-700 \mu$ in diameter (fig. 3).

The endothelium of this type of vessel varies greatly in thickness depending upon the state of contraction of the arteriole. In the nuclear region the endothelium may be as much as $5 \mu$ thick and in other places only $0.2 \mu$. The cells are joined by tight junctions in the usual manner, with one cell interdigitated with or overlapping its neighbor at their point of contact. Pinocytotic vesicles are numerous, but fenestrae are never seen. Microtubules and fine filaments (50-100 $\AA$ in diameter) are visible in the cytoplasm. The filaments and tubules are not arranged in bundles, but for the most part are orientated with the long axis of the cells. A basement membrane underlies the endothelium separating it from the elastica interna.

The elastica interna itself is not a continuous layer, but it consists of a meshwork of elastic fibers and microfibrils about 0.1-

\footnotetext{
2 Paragon C. and C. Company, Inc., 190 Willow Ave., Bronx, New York 10454.

3 The terminology used to describe the various vessels of the vascular tree is not uniform. A summary of some of the terms used is found in the work of Movat and Fernando ('63). The terminology used here is similar to that of Rhodin ('67).
} 
$0.2 \mu$ thick. In vessels larger than $100 \mu$ the elastica interna is usually a complete layer while in vessels of $50 \mu$, or less (fig. 4), elastic components are almost completely absent.

The tunica media of $100 \mu$ arterioles is about $10 \mu$ thick and usually consists of two circumferentially arranged layers of smooth muscle cells. Each cell is enclosed in a basement membrane except at points where the lateral cell membranes are in contact to form a nexus (Dewey and Barr, '62). Numerous fine filaments, microtubules and dense bars, thought to be contractile protein (Rhodin, '67), are evident in the cytoplasm of the smooth muscle cells. The outer layer of the media is well supplied with unmyelinated nerves, which have swellings along their course containing granulated and non-granulated vesicles.

There is no elastica externa in these arterioles. The tunica adventitia varies in thickness from a few tenths of a micron to a few micra. It consists mainly of longitudinally arranged collagen fibers and fibroblasts, which do not appear to be a separate layer surrounding the arteriole, but more like packing material filling the interstices between the vessel and the surrounding structures.

\section{Terminal arterioles}

Vessels of a diameter of less than $50 \mu$ but larger than capillaries are considered to be terminal arterioles (fig. 4). They begin by branching off from arterioles at acute angles and eventually end as capillaries. They are essentially the same as arterioles, but simpler in construction. Their endothelium is the same as the arterioles. An elastica interna is absent and only a single, common basement membrane separates the endothelium from the smooth muscle of the tunica media. Direct membranous contacts between the endothelium and the smooth muscle cells are frequently seen (fig. 4). These myoendothelial junctions are also seen in arterioles at places where there are gaps in the elastica interna, but they are not as common. At these contact points the basement membrane is locally absent. Except for the more numerous myoendothelial contacts and a decrease in thickness, the media of these vessels is like that of the arterioles. It consists of only a single layer of circularly arranged smooth muscle cells. No elastica externa and little or no tunica adventitia are present. The surrounding cells (bile duct cells, fibroblasts, mast cells etc.) are tightly packed directly against the basement membrane of the smooth muscle of the media except in a few places where there are collagen fibers. These vessels appear to be more abundantly supplied with unmyelinated nerves than the arterioles but the nerves themselves are identical.

The terminal arterioles give off numerous capillaries as they traverse the portal space. Eventually these arterioles leave the larger portal areas in company with small distributing veins (fig. 9). By this time their architecture is further simplified, although initially their caliber is only slightly reduced. The smooth muscle coat of these vessels gradually decreases in thickness and eventually becomes incomplete. As more branches are given off, the terminal arteriole is reduced in size to a vessel only slightly larger than a capillary. These vessels finally end by joining either distributing veins or sinusoids.

\section{Precapillaries}

Arising from terminal arterioles in the larger portal spaces are capillaries $(8-10 \mu$ in diameter) which supply the tissues of the portal space. Surrounding the origin of these capillaries are cuffs of smooth muscle (fig. 5) which have been called "precapillary sphincters" (Rhodin, '67). These capillaries and their smooth muscle cuffs constitute a transitional vessel, the precapillary (fig. 6). The sphincters consist of a layer of smooth muscle cells circularly arranged around the origin of the capillary whose axis is at about $90^{\circ}$ to that of the arteriole. The smooth muscle cells of the sphincters have membranous contacts with each other, the media of arteriole and with the endothelium. Nerve endings, containing granulated and nongranulated vesicles, are in membranous contact with the smooth muscle of the sphincters (fig. 5).

Twenty to $30 \mu$ along the origin of the capillary the wall of the sphincter decreases in thickness and the lumen enlarges slightly. Finally the smooth muscle coat becomes incomplete and disappears. From this point on the vessel is a typical capillary 
(fig. 7). Terminal arterioles which follow small distributing veins and have a thin or incomplete media have correspondingly reduced precapillary sphincters, but their general structure is the same.

\section{Capillaries}

The capillaries form a dense network of vessels which ramifies throughout the portal space. Nearly all of these vessels assume a close relationship around the bile ducts to form the periductal or peribiliary plexus (fig. 2). A few capillaries travel more independently through the portal tissue and are not associated with bile ducts, but their structure and terminations are the same as those of the peribiliary plexus.

Capillaries of the portal space are similar to those found in other parts of the body, except for one feature noted below. They have a thin endothelium whose cells are joined by tight junctions. Pinocytotic vesicles, or caveolae intercellulares are abundant, but fenestrae were never seen. Fine filaments and microtubules are numerous in the endothelial cells and are generally arranged with the long axis of the vessel. A prominent basement membrane separates the endothelium from the bile ducts and other tissues of the portal tract. Randomly placed perivascular cells partly surround the capillaries. Occasionally, unmyelinated nerve fibers were seen to be closely associated with the endothelium of large (about $10 \mu$ ) capillaries (figs. 12,13 ). They contained both granulated and nongranulated vesicles and were separated from the endothelium by a basement membrane while on their other surfaces they were covered by Schwann cells. Although they were not common, several were found during the course of this study.

After traveling varying distances in the portal tract the capillaries terminate by joining (1) interlobular veins, (2) terminal distributing veins, (3) sinusoids directly, or (4) sinusoids in common with branches from the portal vein. No vessels of the hepatic arterial system or its branches were found to end near the central veins. All of the capillaries stopped at the periphery of the lobule.

Terminations in interlobular veins. Capillaries may join interlobular portal veins directly (fig. 8), but these connections ap- pear to be quite rare. The capillaries join these veins at acute angles, penetrating the smooth muscle of the media. There are no smooth muscle or perivascular cells around these capillaries near their junction except for the muscle of the interlobular vein itself. Near the opening of the capillary into the vein there are prominent endothelial cells which appear to be "guarding" the entrance into the vein. The nuclei of these endothelial cells bulge into the capillary lumens and in some cases nearly occlude

Abbreviations

BD, Bile duct

BM, Basement membrane

C, Capillary

E, Endothelium

EI, Elastica interna

EN, Endothelial cell nucleus

HA, Hepatic arteriole

ha, Terminal arteriole

KC, Differentiated lining cell (Kupffer cell)

L, Lymphatic vessel

$\mathrm{N}, \mathrm{n}$, Nerve

PC, Precapillary

PCS, Precapillary sphincter

$\mathbf{P V}$, Interlobular portal vein

pv, Terminal distributing vein

$\mathrm{S}$, Sinusoid

SC, Schwann Cell

TA, Tunica adventitia

TJ, Tight junction

TM, Tunica media

Fig. 1 Terminal arborizations of the hepatic artery. Arterioles (HA) and terminal arterioles (ha) give rise to capillaries (C) which surround bile ducts (BD). Capillaries arising from arterioles ( $P C$ ) have well developed precapillary sphincters at their origins, while capillaries from terminal arterioles have only poorly developed sphincters. The periductal capillaries terminate by joining portal veins and/or sinusoids. A few capillaries join larger interlobular veins (PV) and some join terminal distributing veins ( $\mathrm{pv}$ ). Others give rise to sinusoids ( $S$ ) directly (upper right), or to sinusoids in common with the portal vein (upper center). Endothelial cell nuclei (EN) are usually located at or near the junctions of capillaries with other vessels. Sinusoids arising from capillaries are identical in structure to those arising from portal veins. At the periphery of the lobule all sinusoids resemble capillaries, having a complete basement membrane (BM) and unfenestrated endothelium. A short distance jnto the parenchyma they loose their basement membrane, become fenestrated and are true sinusoids. There are numerous lymphatic vessels (L) and unmyelinated nerves $(N, n)$ in the portal tissue. The nerves supply the smooth muscle of arterioles and precapillary sphincters. Occasionally small nerve fibers $(n)$ are found in close relation to endothelial cells. 
them. These cells do not have any special contractile organelles, but exhibit the usual endothelial cell components, microtubules and fine filaments.

Capillaries terminating in distributing veins. Many of the capillaries of the por- tal space end by opening into distributing veins. Most frequently they join veins of $20-50 \mu$ in diameter (figs. 9, 11), but they may also join the larger distributing veins. These distributing veins may travel in parallel with interlobular veins or pass

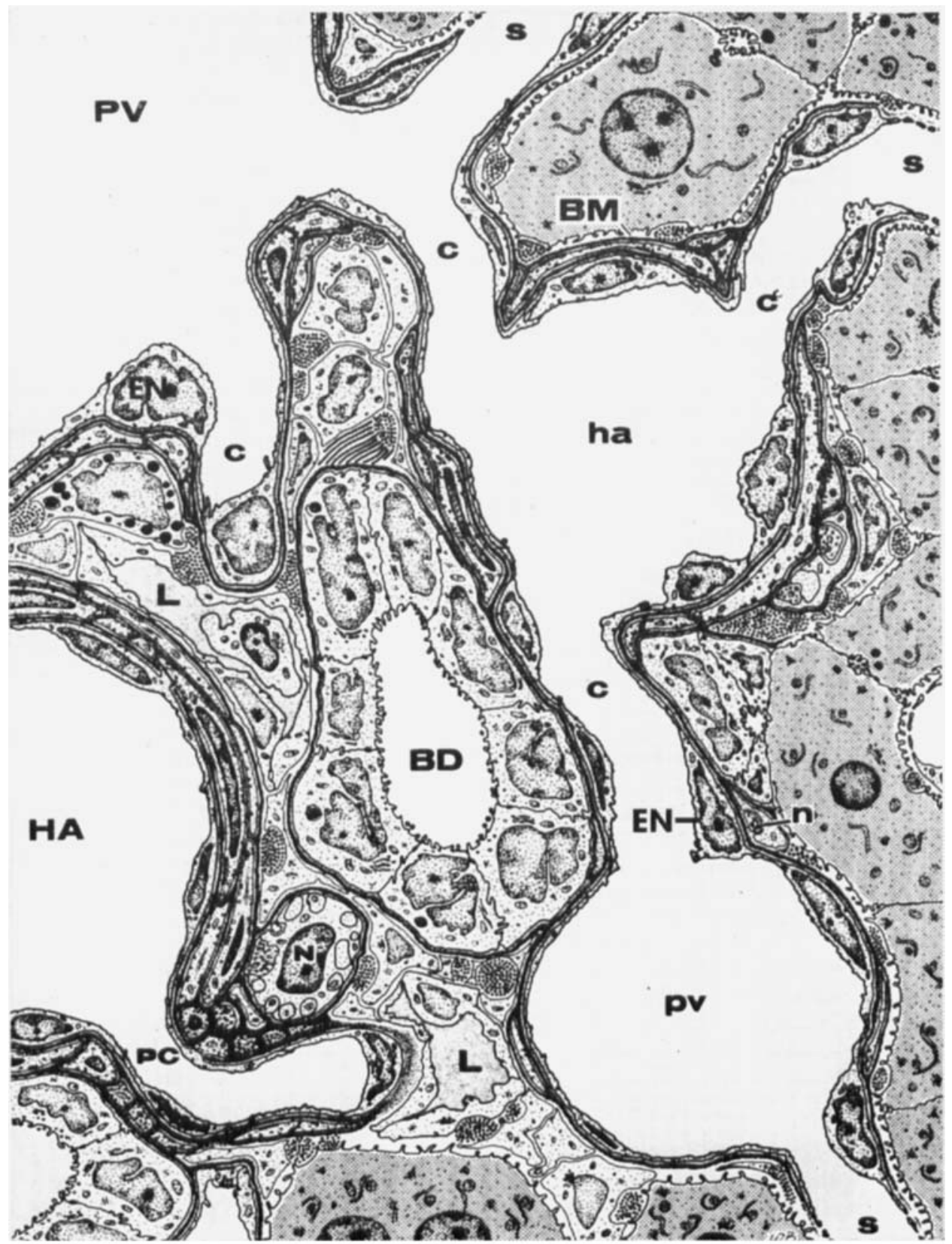

Figure 1 
through the parenchyma alone. The smaller, terminal distributing veins (figs. 9,11 ) are very simple in architecture and, except for their larger size, lack of fenestrae in their endothelium and the presence of a complete basement membrane surrounding them, they would appear to be simply enlarged sinusoids. These veins are near termination and they end by breaking up into sinusoids. Capillaries joining these vessels do not have any specialized sphincter mechanism at their junction with the veins, but there occasionally is a pericyte partly surrounding them. An endothelial cell nucleus at or near the juncture of the two vessels is usually seen (fig. 11). Occasionally unmyelinated nerve fibers containing small vesicles of about $500 \AA$ in diameter (some of which contain dense granules) are seen closely asociated with these endothelial cells (figs. 12, 13). The nerve fibers are separated from the endothelium only by a basement membrane common to them both, but they are separated from the other surrounding tissues by Schwann cells. The cells themselves show no specialized structures other than a few fine filaments and microtubules that would suggest they are capable of contraction.

Capillaries joining sinusoids. The capillaries of the portal tract that do not join portal veins directly give rise to sinusoids at the periphery of the lobule (figs. 14, 15). These sinusoids are identical in structure to those arising directly from the portal veins (figs. 9, 10). They are typical capillaries until they begin to enter the parenchyma. As they enter the parenchyma the basement membrane around them becomes discontinuous and disappears. Fenestrae appear in the sinusoidal lining cells as soon as the basement membrane is gone and the vessels become true sinusoids. At the point where the fenestrae begin it is impossible to tell for certain whether the capillary endothelium actually becomes fenestrated or that the capillary endothelial cell ends and the adjacent lining cell is the fenestrated one. The latter would appear to be the case.

There are no specialized sphincters at the beginning of these sinusoids other than the endothelial cells themselves. The orifice at the beginning sinusoid at the periphery of the lobule is nearly always buttressed with bundles of collagen, so it would seem that the only way the opening could easily be made smaller would be by the bulging of the endothelium into the lumen to occlude it.

The capillaries of the portal tract also give rise to sinusoids in common with twigs coming from distributing veins. Except for a slight dilatation where the portal vein twig and the capillary meet the sinusoids thus formed are identical to the ones mentioned above.

\section{DISCUSSION}

Figure 1 summarizes the various endings of the hepatic artery of the rat and their structure. Because of the technical difficulties involved in doing serial sections of large numbers of portal areas at the electron microscope level, this study is not suited to determine the frequency of occurrence of the types of endings observed. It was possible, however, to find examples of the most common endings which have been described in injection (Mitra, '66) and transillumination investigations (McCuskey, '66). These endings, diagrammatically represented in figure 1 , are: (a) terminations of capillaries in interlobular veins, (b) terminations of capillaries in distributing veins, (c) terminations of capillaries in sinusoids directly and (d) terminations of capillaries in sinusoids in common with twigs off from the portal vein.

No evidence was found to support the existence of "arterial sinusoids" described in earlier studies (Wakim and Mann, '42; Elias, '49; Senevirante, '49; Elias and Petty, '53; Riedel and Moravec, '59; Lee et al., '60; Tajiri, '60). The descriptions of "arterial sinusoids" might be explained by the fact that lobules are frequently poorly defined (Hase and Brim, '66; Bloom and Fawcett, '68) and that capillaries thought to be within the lobule are in reality at the periphery of an irregular lobule and traveling with a small terminal distributing vein. Many of the terminal distributing veins are very small $(15-20 \mu)$ and except for their slightly larger size are impossible to distinguish from sinusoids at the level of light microscopy. In electron microscope preparations these terminal distributing veins can be distinguished from sinusoids because they have an unfenestrated endothe- 
lium and a complete basement membrane around them. In contrast, sinusoids lack a basement membrane and are fenestrated. This view is compatible with that of recent reports on the vasculature of the liver (McCuskey, '66; Mitra, '66; Hase and Brim, '66).

From the present investigation it appears that "inlet sphincters," "outlet sphincters" and "intersinusoidal sphincters" described in transillumination studies are, as McCuskey ('66) suggests, contractile endothelial or reticulo-endothelial cells. But at the electron microscope level it is possible to see differences between the cells functioning as "inlet" and "outlet sphincters" on the one hand and the "intersinusoidal sphincters" on the other. The cells forming "inlet" and "outlet sphincters" at the ends of the sinusoid are extensions of the endothelium of the portal and central veins or capillaries into the parenchyma (figs. 9, 10). Like other endothelial cells, the cells acting as "inlet" and "outlet" sphincters are unfenestrated and have a complete basement membrane around them. The cells considered to be "intersinusoidal" sphincters are entirely within the lobule itself. These lack a basement membrane, are fenestrated and many are phagocytic. These cells should be called undifferentiated lining cells if they are not presently, but potentially phagocytic, and differentiated lining cells (von Kupffer cells) if they are phagocytic. Both of these types are more properly considered to be reticulo-endothelial cells.

Other cells in the lobule may also perform sphincter-like functions (Burkel, '68). These cells are either fibroblasts or "fat storage cells" (Ito and Nemoto, '52) and lie in the space of Dissé. Regardless of the type of cell involved all of the cells which may act as sphincters possess structural elements usually associated with contraction, i.e. fine filaments and microtubules (Majno et al., '67). Presumably any of the cells lining sinusoids could act as sphincters by bulging into the lumen of the vessel to occlude it. This may be a passive phenomenon or it may be actively controlled by local autoregulation (McCuskey, '66).

The significance of the small unmyelinated nerve fibers in close association with the endothelial cells of some periductal capillaries (figs. 12, 13) is uncertain. Innervation of endothelium has not been described (Majno, '65), although Tanikawa ('68) has shown unmyelinated nerve fibers in close relation to the endothelium of portal veins in the liver and Majno et al. ('69) have noted profiles similar to nerves in association with vascular pericytes. These nerve fibers are separated from the endothelium only by a basement membrane common to them both. They seem to be more intimately related to the endothelium than to any other structures and are covered by Schwann cells on all surfaces except that adjacent to the endothelum. Since the ability of endothelial cells to contract is fairly well documented (Majno et al., '67), it may be that these cells are acting as sphincters in these capillaries (particularly at their junctions with portal veins and sinusoids) and are under the influence of autonomic nerves. Sphincters in the peribiliary capillaries at their juncture with branches of the portal venous system have been described in living animals by McCuskey ('66), but it is unclear from his work just what kind of cells are involved. Smooth muscle cells have not been seen around these capillaries except at their origin from arterioles and perivascular cells are only irregularly placed along the vessels. It may be that the constriction of endothelial cells under the control of autonomic nerves is responsible for controlling the flow of blood here, but this remains to be proven.

\section{LITERATURE CITED}

Bloch, E. H. 1940 Some actions of adrenaline chloride and acetyl-beta-methyl choline chloride of finer vessels of liver lobules. Anat. Rec., 76: 7.

1955 The in vivo microscopic vascular anatomy and physiology of the liver as determined with the quartz-rod method of transillumination. Angiology, 6: 340-349.

Bloom, W., and D. W. Fawcett 1968 A Textbook of Histology. 9th ed. W. B. Saunders Co., Philadelphia.

Burkel, W. E. 1968 Unpublished results.

Burkel, W. E., and F. N. Low 1966 The fine structure of rat liver sinusoids, space of Disse and associated tissue space. Am. J. Anat., 118: $769-784$.

Dewey, M. M., and L. Barr 1962 Intercellular connection between smooth muscle cells: The Nexus. Science, 137: 670-672. 
Elias, H. 1949 A re-examination of the structure of the mammalian liver. II. The hepatic lobule and its relation to the vascular and biliary systems. Am. J. Anat., 85: 379-456.

Elias, H., and D. Petty 1953 Terminal distribution of the hepatic artery. Anat. Rec., 116: 9-17.

Hase, T., and J. Brim 1966 Observation on the microcirculatory architecture of the rat liver. Anat. Rec., 156: 157-174.

Irwin, J. W., and J. MacDonald 1953 Microscopic observations of the intrahepatic circulation of living guinea pigs. Anat. Rec., 117: 115.

Ito, T., and M. Nemoto 1952 Uber die kupfferschen sternzellen und die "fettspeicherungszellen" ("fat storing cells") in der blutkapillarenwand der menschlichen leber. Okajimas Folia Anat. Japan, 24: 243-258.

Karnovsky, M. J. 1965 A formaldehyde-glutaraldehyde fixative of high osmolarity for use in electron microscopy. J. Cell Biol., 27: 137A138A.

Knisely, M. H. 1936 A method of illuminating living structure for microscopic study. Anat. Rec., 64: 499-524.

Knisely, M. H., F. Harding and H. Debacker 1957 Hepatic sphincters. Science, 125: 1023-1026.

Lee, Y. B., H. Elias and I. Davidsohn 1960 Vascular pattern in the liver of the mouse. Proc. Animal Care Panel, 10: 25-32.

Luft, J. H. 1961 Improvements in epoxy resin embedding methods. J. Biophys. Biochem. Cytol., 9: $409-414$.

Majno, G. 1965 Ultrastructure of the vascular membrane. In: Handbook of Physiology, section 2 , volume 3 . W. F. Hamilton and P. Dow, eds. Washington D.C., Am. Physiol. Society, pp. 2293-2375

Majno, G., V. Gilmore and M. Leventhal 1967 On the mechanism of vascular leakage caused by histamine-type mediators: A microscopic study in vivo. Cir. Res., 21: 823-832.

Majno, G., S. Shea and M. Leventhal 1969 Endothelial contraction induced by histamine-type mediators. An electron microscopic study. J. Cell Biol., 42: 647-673.
McCuskey, R. S. 1966 A dynamic and static study of hepatic arterioles and hepatic sphincters. Am. J. Anat., 119: 455-478.

Mitra, S. K. 1966 The terminal distribution of the hepatic artery with special reference to arterio-portal anastomosis. J. Anat., 100: 651663.

Movat, H. Z., and N. V. P. Fernando 1963 The fine structure of the terminal vascular bed. I. Small arteries with an internal elastic lamina. Exp. and Molec. Path., 2: 549-563.

Palay, S. L., S. M. McGee-Russell, S. Gordon and M. A. Grillo 1962 Fixation of neural tissues for electron microscopy by perfusion with soIutions of osmium tetroxide. J. Cell Biol., 12: $385-410$.

Reynolds, E. S. 1963 The use of lead citrate at high $\mathbf{p H}$ as an electron opaque stain in electron microscopy. J. Cell Biol., 17: 208-212.

Rhodin, J. A. G. 1967 The ultrastructure of mammalian arterioles and precapillacy sphincters. J. Ultrastructure Res., 18: 181-223.

Riedel, J., and R. Moravec 1959 Das system der pfortader und der leberarterie in der leber der ratte. Anat. Anz., 107: 99-110.

Sabatini, D. D., K. Bensch and R. J. Barrnett 1963 Cytochemistry and electron microscopy: The preservation of cellular ultrastructure and enzymatic activity by aldehyde perfusion. $J$. Cell Biol., 17: 19-58.

Senevirante, R. D. 1949 Physiological and pathological responses in blood vessels of liver. Quart. J. Exp. Physiol., 35: 77-110.

Tajiri, S. 1960 The terminal distribution of the hepatic artery. Acta Med. Okayama, 14: 215225.

Tanikawa, K. 1968 Ultrastructural aspects of the liver and its disorders. Springer-Verlag, Berlin, pp. 54, 55.

Wakim, K. G. 1944 The effect of certain substances on the intrahepatic circulation of blood in the intact animal. Amer. Heart J., 27: 289300.

Wakim, K. G., and F. C. Mann 1942 The intrahepatic circulation of blood. Anat. Rec., 82: $233-253$. 
PLATES 
PLATE 1

EXPLANATION OF FIGURES

2 A portion of a typical large portal area. The portal space contains a large interlobular vein (PV) about $600 \mu$ in diameter and two small distributing veins ( $\mathrm{pv}$ ). A branch of the hepatic artery ( $\mathrm{HA}$, right) closely follows the interlobular vein and it gives rise to an arteriole (HA, left) and a terminal arteriole (ha). The arterioles give rise to the numerous capillaries (C) adjacent to the bile ducts (BD). The precapillary (PC) is a transitional vessel between the arterioles and capillaries. Numerous unmyelinated nerves $(N)$ and lymphatic vessels (L) are also found in the portal area. The following figures show details of some of the vessels in this micrograph. $\times 840$.

3 Large arteriole. This micrograph shows a portion of the large arteriole (about $100 \mu$ in diameter) on the right side of figure 2. The endothelium has the usual pinocytotic vesicles (arrow) and tight junctions (TJ) between its cells. Elastic fibers and microfibrils make up the nearly complete elastica interna (EI). The tunica media (TM) of the vessel consists of two layers of smooth muscle, the cells of which are enclosed in basement membranes (BM). The tunica adventitia (TA) is variable in thickness and contains some unmyelinated nerves $(\mathrm{N})$ and their enclosing Schwann cell. $\times 7000$. 


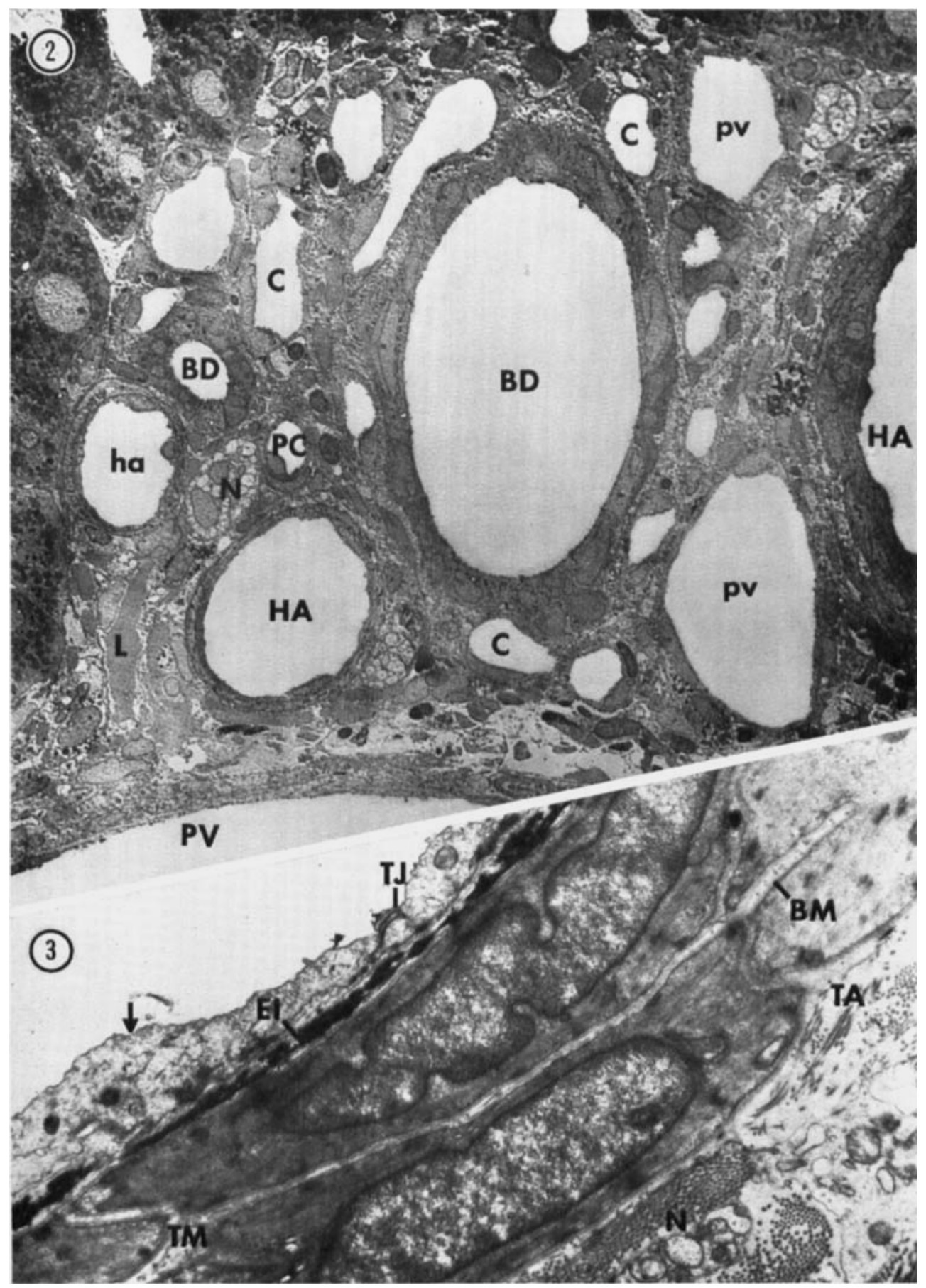


PLATE 2

EXPLANATTON OF FIGURES

4 Terminal arteriole. This vessel is a branch of the arteriole in figure 3 and is also seen (ha) in figure 2 . It is similar to an arteriole, but simpler in architecture. The endothelium (E) is typical. There is no elastica interna, but it is represented by a common basement membrane (BM) between the endothelium and tunica media (TM). The media is reduced to one layer of smooth muscle cells and it receives projections from the endothelium (inset) to form myoendothelial contacts (arrow). The surrounding cells of the portal space are tightly packed against the media with only a basement membrane intervening. Numerous unmyelinated nerves (N) supply these vessels. $\times 3600$; inset, $\times 7100$.

5 Precapillary sphincter. This micrograph shows the origin of a capillary (C) off from an arteriole (HA) about $60 \mu$ in diameter. At these places smooth muscle cells of the arteriole media (TM) form a cuff of cells surrounding the endothelium of the capillary. The cuff is called a precapillary sphincter (PCS) and the sphincter and endothelium together constitute a precapillary. The smooth muscle cells of the sphincter are in membranous contact with each other, with the endothelium (arrows) and with unmyelinated nerve fibers (N). $\times 4300$. 

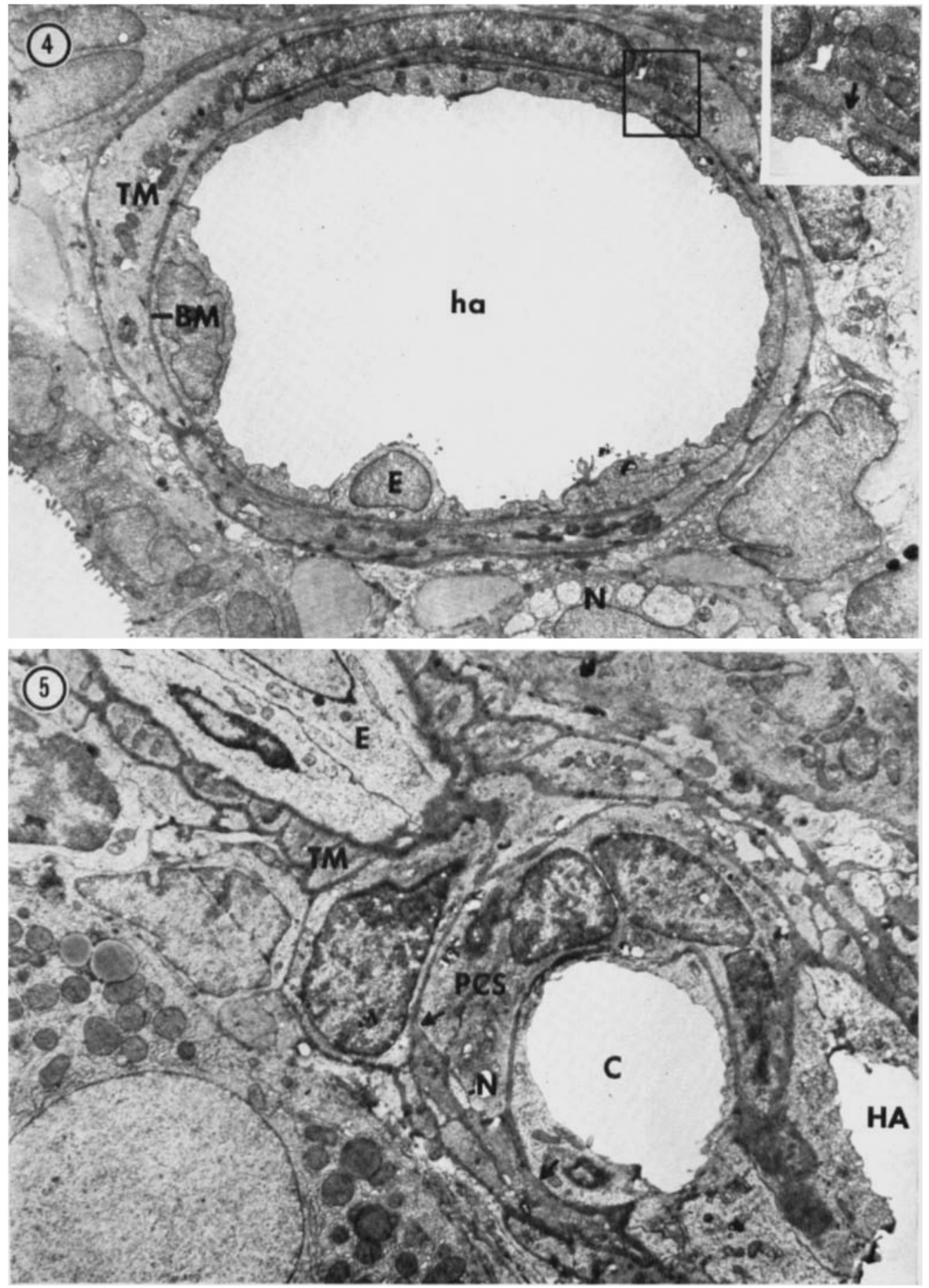
PLATE 3

EXPYANATION OF FIGURES

6 Precapillary. The precapillary (PC) looks very much like a miniature arteriole. It has a complete smooth muscle coat at this point, but it disappears $20-30 \mu$ from the origin of the vessel. The smooth muscle is well supplied with unmyelinated nerves $(N) . \times 3800$.

7 Periductal capillary. The hepatic arterioles give rise to the rich network of capillaries of the portal space, the periductal plexus. They are like the capillaries in other parts of the body, but are in very close association to the bile ducts (BD). $\times 17,000$.

8 Junction of a periductal capillary with an interlobular vein. The capillary (C) joins the vein (PV) at an acute angle. There is no sphincter surrounding this end of the capillary other than the smooth muscle of the interlobular vein. A large endothelial cell nucleus (EN) "guards" the junction. Connections such as this between the periductal plexus and interlobular veins are relatively rare. $\times 5500$. 

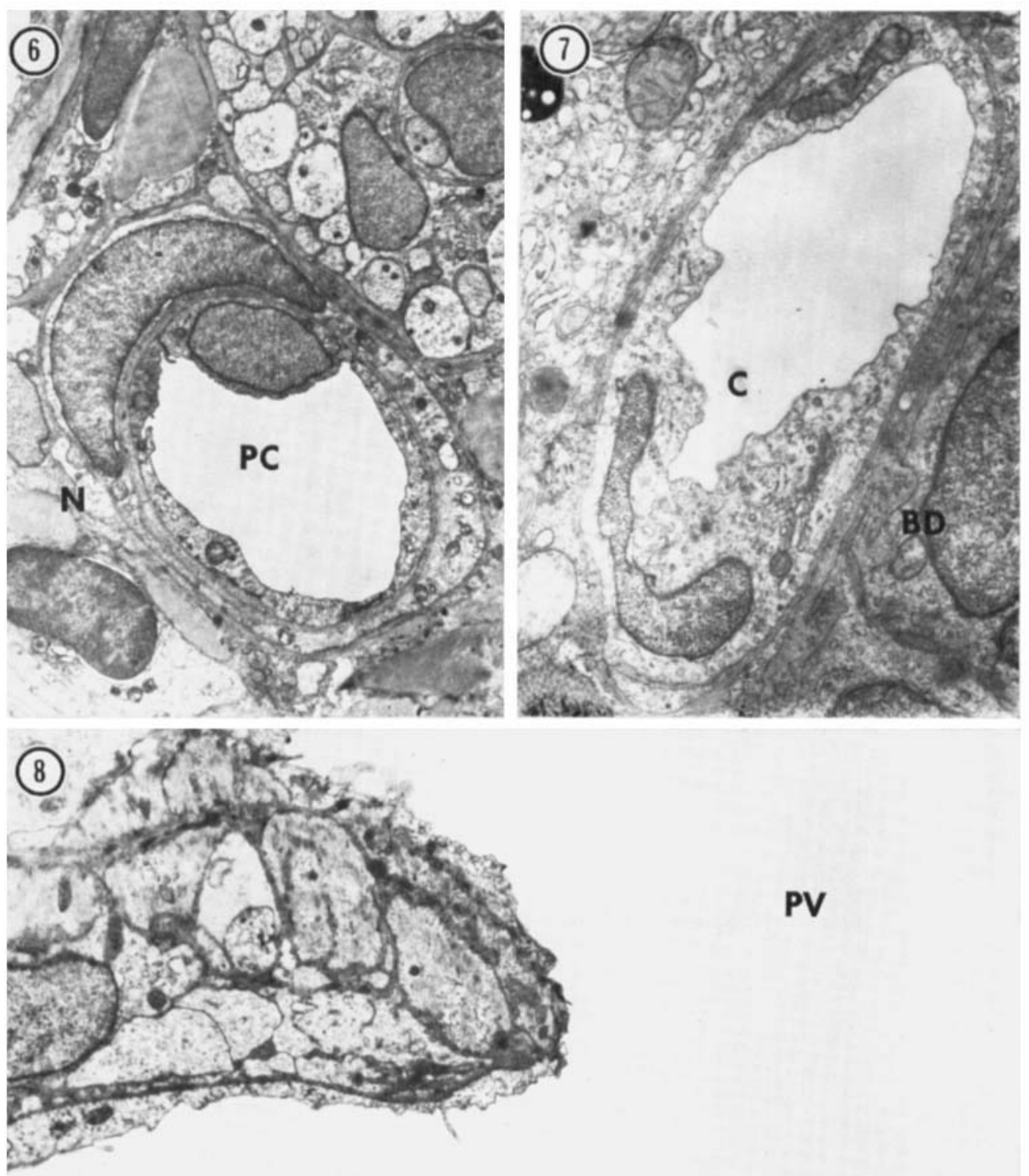

\section{PV}

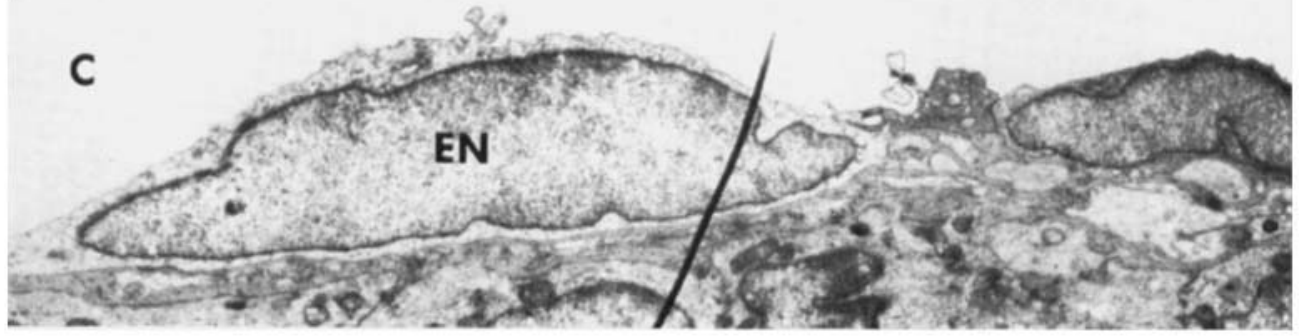


PLATE 4

EXPLANATION OF FIGURES

9 A small portal area. This portal area consists of a distributing vein (PV) giving rise to a large sinusoid (S), a bile duct (BD), a small terminal branch of the arterial system (ha) and a terminal distributing vein ( $p v$ ). The beginning of the sinusoid is "guarded" by two large endothelial cell nuclei (EN) on either side of the vessel (see figure 10 for more detail). The arterial system is represented by a vessel (ha) only slightly larger than a capillary at this point. The terminal distributing vein is about to be joined by a periductal capillary (arrow). From serial sections it was determined that the capillary arose from the terminal arteriole (ha). Note the large endothelial cell nucleus near the junction of the two vessels. Figure 11 shows more details of this area. $\times 840$.

10 Endothelial cell at the origin of a sinusoid. This endothelial cell (E) is found at the origin of the sinusoid (S) off from the portal vein (PV) of figure 9 (rectangle). The portal vein is to the left. This cell is continuous with and identical to the endothelium of the portal vein. It is unfenestrated and is underlain by a basement membrane (BM). Farther into the parenchyma along the course of this vessel the cell becomes fenestrated and the basement membrane disappears (arrow). $\times 14,000$. 

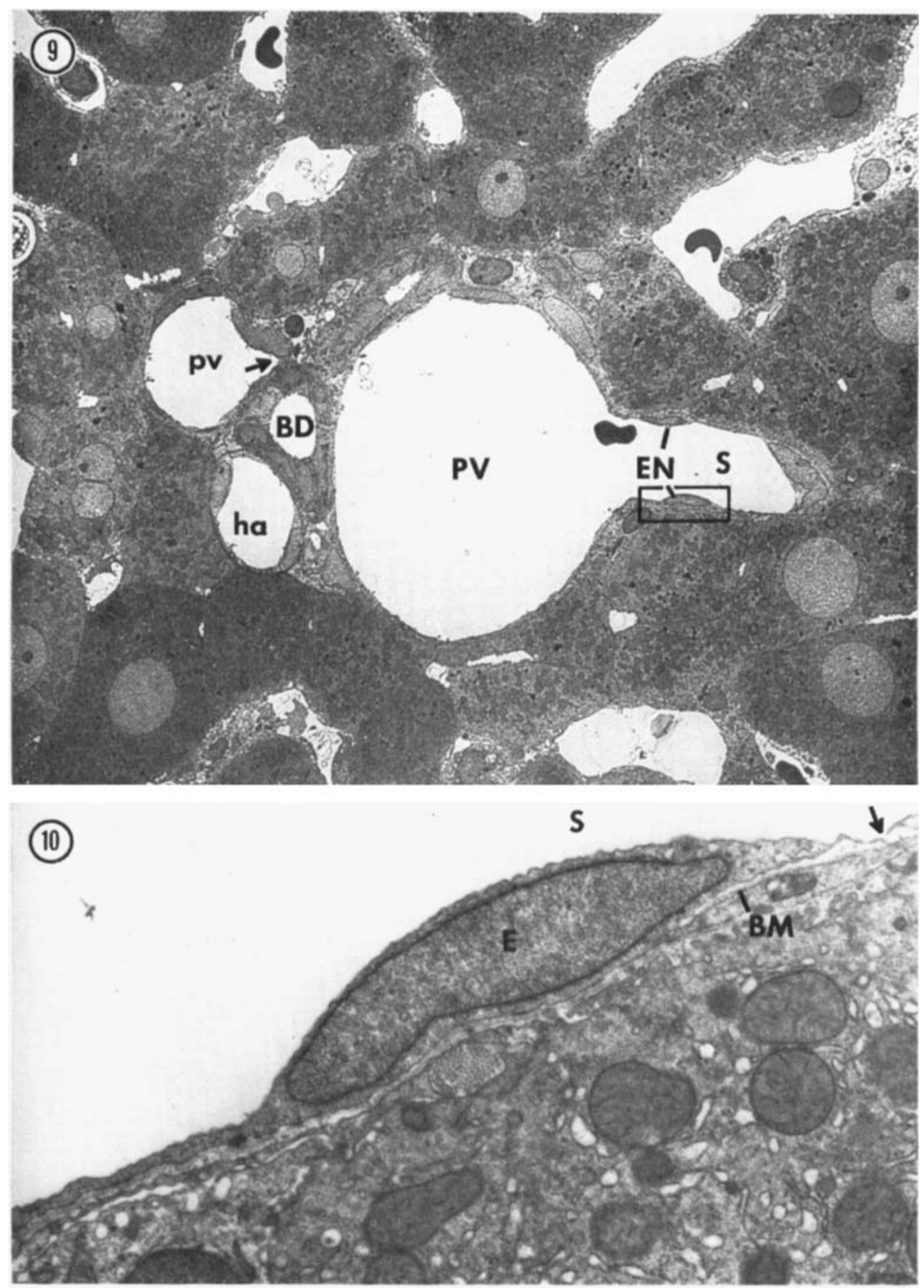
PLATE 5

EXPLANATION OF FIGURES

11 Periductal capillary joining a terminal distributing vein. This section is a few microns from that of figure 9. The terminal arteriole (ha) had previously given rise to the periductal capillary (C) which is now joining the terminal distributing vein ( $p v$ ). The terminal arteriole is only slightly larger than a capillary and has thin, incomplete smooth muscle layer around it. The terminal distributing vein is only slightly larger than a sinusoid, but it has a continuous unfenestrated endothelium surrounded by a complete basement membrane. A large endothelial cell encircles the capillary at its junction with the terminal distributing vein. Figure 12 shows more detail of this capillary. $\times 4600$.

12 Periductal capillary before joining terminal distributing vein. This section shows the periductal capillary (C) of figure 11 just before it joins the terminal distributing vein (pv). A large endothelial cell nucleus (EN) surrounds the capillary at the junction. Two unmyelinated nerve fibers $(N)$ are closely related to the endothelial cell (see fig. 13). $\times 5600$.

13 Detail of unmyelinated nerve and endothelium from figure 12 (rectangle). The unmyelinated nerve fibers ( $N$ ) contained both granulated and ungranulated vesicles. They are separated from the endothelium ( $E$ ) only by a common basement membrane (BM), but are covered on all other surfaces by a Schwann cell (SC). $\times 31,000$. 

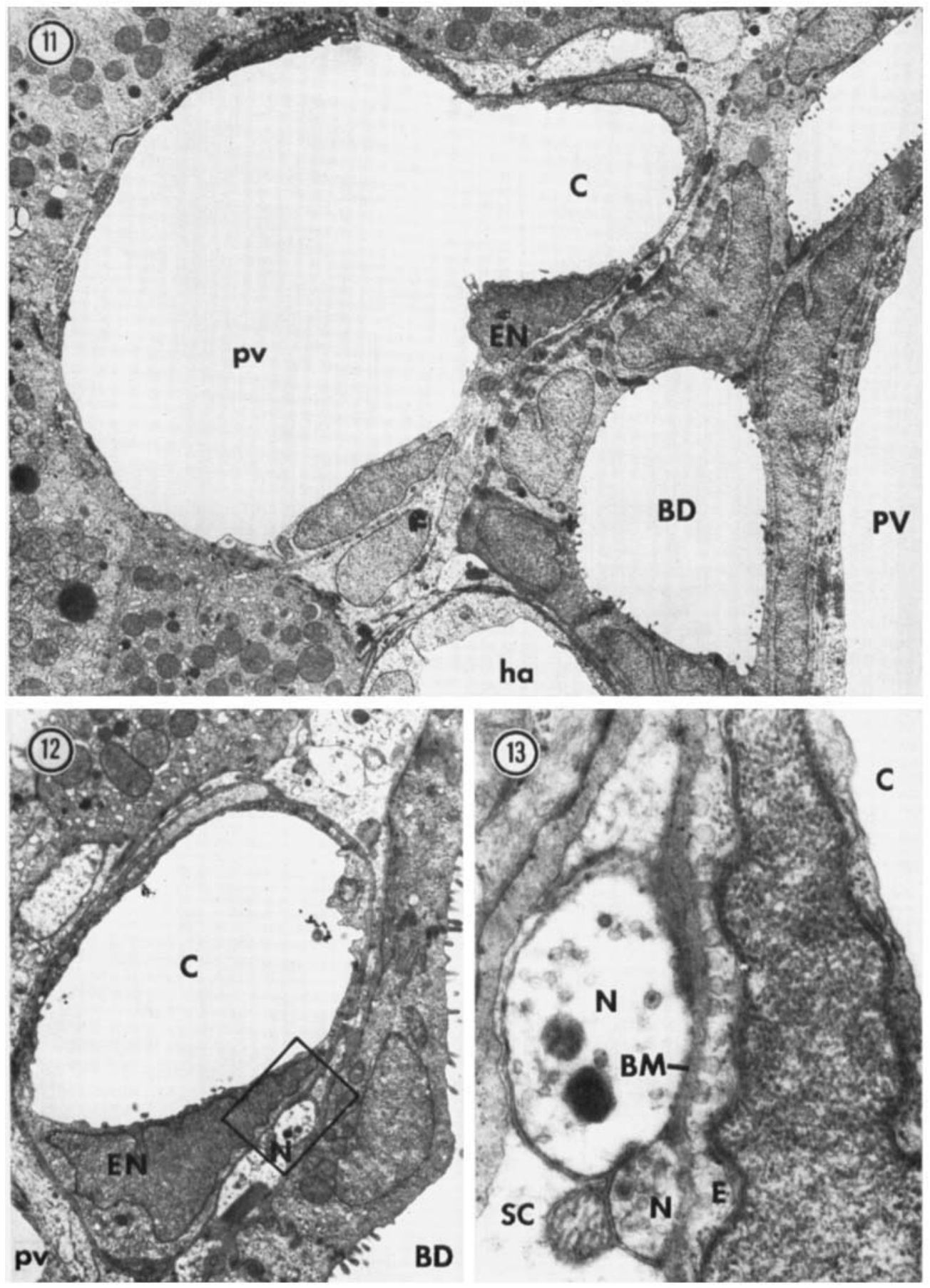
PLATE 6

EXPLANATION OF FIGURES

14 Periductal capillary ending in a sinusoid. The periductal capillary (C) leads into the sinusoid (S). Near the hepatic arteriole (ha) from which it took origin, the vessel is an unfenestrated capillary with a complete basement membrane (inset, right). A short distance into the parenchyma the vessel loses its basement membrane, fenestrae begin to appear (arrow) and it becomes a typical sinusoid such as figure 15. $\times 3900$ (inset, $\times 12,000$ ).

15 Sinusoid. This sinusoid (S) is similar to the vessel at the left of figure 14, but cut in cross section. It displays typical sinusoid features such as fenestrae and lack of basement membrane (arrow). The differentiated lining cell (KC) contains some phagocytosed material. Occasionally, these cells span the sinusoid and apparently occlude it to stop blood flow. $\times 4500$. 


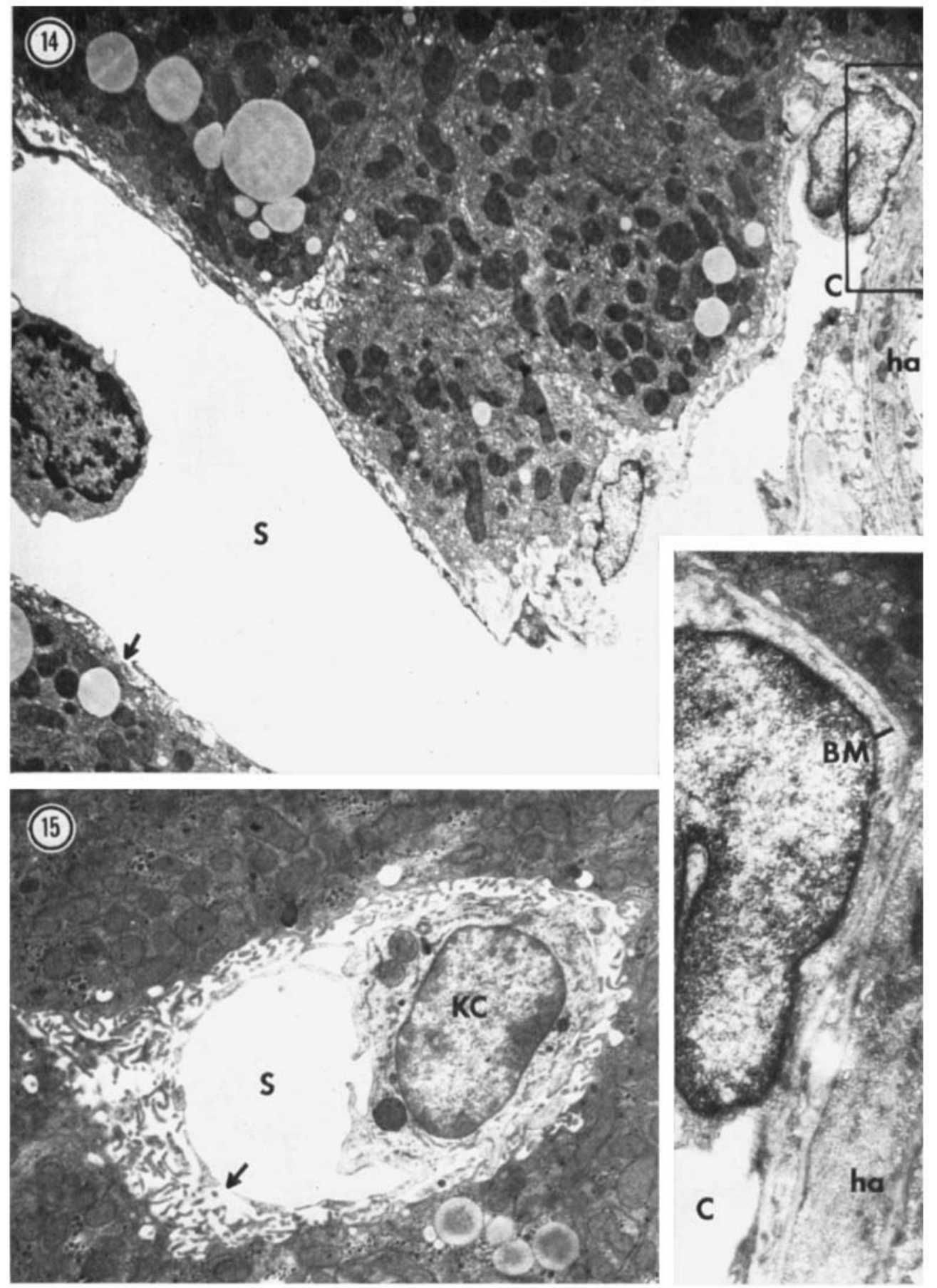

